\title{
BMJ Open POP-Brazil study protocol: a nationwide cross-sectional evaluation of the prevalence and genotype distribution of human papillomavirus (HPV) in Brazil
}

\author{
Eliana Marcia Wendland, ${ }^{1,2}$ Juliana Caierão, ${ }^{1}$ Carla Domingues, ${ }^{3}$ \\ Ana Goretti Kalume Maranhão, ${ }^{3}$ Flávia Moreno Alves de Souza, ${ }^{4}$ \\ Luciano Serpa Hammes, ${ }^{1}$ Maicon Falavigna, ${ }^{1,5}$ Juliana Balbinot Hilgert, ${ }^{6}$ \\ Fernando Neves Hugo, ${ }^{6}$ Marina Bessel, ${ }^{1}$ Luisa Lina Villa, ${ }^{7}$ \\ Adele Schwartz Benzaken ${ }^{4}$
}

To cite: Wendland EM, Caierão J, Domingues C, et al. POP-Brazil study protocol: a nationwide cross-sectional evaluation of the prevalence and genotype distribution of human papillomavirus (HPV) in Brazil. BMJ Open 2018;8:e021170. doi:10.1136/ bmjopen-2017-021170

- Prepublication history for this paper is available online. To view these files, please visit the journal online (http://dx.doi. org/10.1136/bmjopen-2017021170).

Received 15 December 2017

Revised 24 April 2018

Accepted 4 May 2018
Check for updates

For numbered affiliations see end of article.

Correspondence to Dr Eliana Marcia Wendland; eliana.wendland@hmv.org.br

\section{ABSTRACT}

Introduction Human papillomavirus (HPV) is associated with the development of genital warts and different types of cancer, including virtually all cervical cancers and a considerable number of penile, anal and oropharyngeal cancers. Data regarding the prevalence of HPV infection in Brazil are limited and fragmented. We aim to determine HPV prevalence in sexually active women and men aged 16-25 years and to investigate regional differences in virus prevalence and types.

Methods and analysis This is a nationwide, multicentric, cross-sectional, prospective study that will include participants aged 16-25 years from all Brazilian capital cities. Recruitment will occur in primary health units by trained health professionals who will be responsible for collecting biological samples and interviewing the volunteers. After signing informed consent, all participants will answer a questionnaire that will collect sociodemographic and behavioural data. All samples will be processed in a certified central laboratory, and strict quality control will be performed by many different procedures, including double data entry, training and certification of primary care health professionals responsible for data collection, simulation of interviews, and auditing and monitoring of visits. The sample size will be standardised based on the population distribution of each capital using SAS and R statistical software.

Ethics and dissemination The project was approved by the research ethics committee of the main institution and the corresponding ethics committees of the recruitment sites. This will be the first Brazilian nationwide study to determine overall HPV prevalence and to examine regional differences and social, demographic and behavioural factors related to HPV infection. Critical analysis of the study results will contribute to epidemiological knowledge and will set a baseline for future evaluation of the impact of the National HPV Vaccination Program.

\section{INTRODUCTION}

According to the WHO, more than 528000 incident cases and approximately 270000 deaths due to cervical cancer occur annually
Strengths and limitations of this study

Representativeness of all regions of Brazil.

- Establishment of a baseline to evaluate the effectiveness of the human papillomavirus vaccination programme in Brazil.

- Recruitment done in primary care units, inviting all people within the age range living in the area and not over-representing risk-factor groups.

- Only the population that uses the primary care units from the public health system will be included but that consists of $71 \%$ of the Brazilian population.

worldwide, $85 \%$ of which occur in low-income and middle-income countries. ${ }^{1}$ Virtually all these cancers are preceded by infection with high-risk oncogenic human papillomaviruses (HR-HPVs), ${ }^{2}$ and HPV types 16 and 18 are associated with $70 \%$ of these cases. ${ }^{34}$ In Brazil, the National Institute of Cancer estimated that there were 16340 new cervical carcinoma cases in 2016 , with a $33.23 \%$ mortality rate. ${ }^{5}$

HR-HPVs are also responsible for $63 \%$ of penile cancers, $89 \%$ of anal cancers and $72 \%$ of oropharyngeal cancers in men. ${ }^{67}$ Indeed, these viruses are now recognised as the major cause of the observed rising incidence rates of oropharyngeal squamous cell carcinoma; indeed, the primary risk factor for developing this cancer is oral HPV infection. ${ }^{8}$

Most sexually active individuals will be infected by HPV at some point in their lives. ${ }^{910}$ In Brazil, the prevalence of HPV infection in women with normal cervical cytology varies from $2.3 \%$ to $55.4 \% .{ }^{11}{ }^{12}$ Considerable variability in HPV prevalence rates for other body sites, such as the penile epithelium and the oral cavity, is also observed in Brazil. ${ }^{11} 12$ 
These data originated from studies that were heterogeneous with regard to methodology and/or the study population. In addition, virtually all studies to date were geographically restricted ${ }^{13-17}$ and/or focused on specific groups of individuals, such as HIV-positive, pregnant and immunosuppressed patients. ${ }^{18-20}$ Moreover, some of those studies recruited participants from clinics specialising in sexually transmitted infections (STIs), resulting in selection bias and inflation of the estimated prevalence. ${ }^{13-20}$

Vaccination against HPV is being performed in many countries, and it is a method of primary prevention of cancer and HPV infection. ${ }^{9}$ Since 2014, the Brazilian Ministry of Health has provided a quadrivalent (types 6, 11,16 and 18) vaccine for women aged 9-14 years, with a schedule of 0 and 6 months. In January 2017, Brazil was the first country in Latin America to extend vaccination to men (11-14 years of age).$^{21}$ Baseline data of HPV prevalence before vaccinations are vital in order to establish the impact of vaccination on the distribution of HPV types.

As far as we know, this is the first study presenting a nationwide scope with uniform methodology to evaluate the Brazilian prevalence of HPV and its types, as well as to compare the prevalence among specific geographical regions that present marked differences in social, demographic, cultural and economic characteristics. In addition, the study will describe the social, demographic, economic and behavioural factors associated with HPV positivity.

\section{OBJECTIVES}

The primary objective of the study is to determine the prevalence of HPV in women and men aged 16-25 years in Brazil, evaluating the most prevalent types and possible differences between regions and risk factors associated with positivity to establish a baseline for evaluation of vaccine effectiveness.

\section{Secondary objectives}

- To determine the prevalence of HPV, high-risk types, genital warts and the associated sexual behaviours.

- To evaluate the coinfection of HPV-HIV in the young population that uses the public health system.

- To investigate the beliefs and knowledge about HPV infection and vaccination in a young population.

- To evaluate the association of other STIs, such as syphilis, with the presence of HPV.

- To evaluate the herd effect in a sample of unvaccinated men.

\section{METHODS AND ANALYSIS \\ Design}

We will use a cross-sectional, nationwide, multicentric design to establish a baseline dataset that will enable prevalence comparison over time.

\section{Recruitment and sample size}

Beginning in January 2017, sexually active women and men aged 16-25 years who use the public health system in all 26 Brazilian capitals plus the Federal District of Brasilia across the five geographical regions will be recruited through public primary care units.

Because endocervical collection is not recommended during pregnancy in Brazil and/or to avoid selection bias, the following exclusion criteria will be applied: pregnant women, those who delivered a baby in the last 3 months, those who have undergone a hysterectomy or trachelectomy and those who have ever had cervical intraepithelial neoplasia grade 2 or higher will be considered ineligible for the study.

There will be no exclusion based on HPV vaccination status, but we will investigate whether the participants have been vaccinated. Since the vaccination programme was launched in 2014 for women who were 9-13 years of age and our study population is among $16-25$ years old, we believe that most of the volunteers will be not have been vaccinated.

The primary care units in each city were selected based on their representativeness of the health districts and based on their resources to collect and store oral samples. Most of these units have a defined territory and a list of all people within the age range. When the list is available, all participants will be invited. When the list is not available, participants will be recruited by different approaches: (1) eligible individuals who came to the unit for any reason not associated with the main study's outcome, (2) personal invitation by the community health agents and (3) invitation by nurses in the school-based health promotion programme. The study sample size (7935 individuals) was calculated to detect differences of $5 \%$ in HPV prevalence between regions with a power of $80 \%$ and an alpha error of $5 \%$ for women using an estimate of $30 \%{ }^{22}$ for the prevalence of HPV in Brazil. This sample size will allow an estimate with an error between $1.6 \%$ and $5.7 \%$, according to sex.

The sample size will be purposely equal in all regions (1587 individuals) to maximise the diversity in less-populated areas and will be standardised by the Brazilian population of each region during analyses.

\section{Measurements \\ Sociodemographic variables}

Participants will be asked to answer questions regarding age, gender, race/skin colour, household characteristics, relationship status, educational level, occupational status, last-month family income and the number of people dependent on this income.

\section{Smoking, alcohol and other drugs}

Questions concerning current and past smoking habits, including quantity and frequency, will be asked of the participants. Moreover, the interview includes questions about the use of alcohol and other drugs such as marijuana, cocaine, ecstasy, crack and the abuse of morphine or other licit and illicit drugs. 


\section{Sexual and reproductive health}

Participants will be asked to supply details about their age at first sexual intercourse, their involvement in same sex relationships, their use of condoms, the number of sexual partners, their sexual practices and the presence of any genital symptoms during or after sexual intercourse such as pain or bleeding.

Their age of menarche, the number of pregnancies and deliveries, the number of abortions, their use of contraceptive methods and the occurrence of STIs will be asked of participants to evaluate their sexual health. All suspicious HPV-related lesions will be photographed for future evaluation.

\section{Knowledge about HPV, vaccination and screening tests}

We will measure how much participants know about HPV and vaccination (when and who must receive the vaccine). The questionnaire is based on Saulle et al. ${ }^{23}$ Moreover, participants will be asked if they know about Pap smears, if they have already undergone the examination and if so, whether any alteration have ever been observed.

\section{HPV frequency and type}

We will measure the proportion of the sample that is infected by HPV, and we will look at the prevalence of high-risk types and the most prevalent types in the genital and oropharyngeal sites. Additionally, we will investigate the presence of chronic lesions (lasting 15 days or more) in the oral cavity that are related to the presence of HPV.

\section{Outcomes}

The nationwide prevalence of HPV in the genitals is the major outcome. Secondary outcomes include the prevalence of specific HPV types, the comparison of prevalence between Brazilian regions and the prevalence of HR-HPVs and low risk-HPVs.

\section{Procedures}

All individuals will respond to a standardised interview based on validated instruments. ${ }^{23-26}$ After the interview, samples from the oral cavity and cervical or penile/scrotal sites will be collected from each participant.

An online platform for data entry will be used by primary care professionals to add participant data, biological sample information and photographs. The same platform will be used for study process control and to allow availability of the results to primary care health professionals. Participants will have access to an external webpage where they will be able to find information about their results, as protected by a password provided during interview. In cases in which HR -HPV infection is detected, those participants will be asked to return to the primary care unit to be informed about the result.

Oral samples will be obtained through mouthwash and gargle cycles by using $10 \mathrm{~mL}$ of a standardised commercial mouthwash. The samples will be obtained in 3 cycles of $5 \mathrm{~s}$ each and will be stored in a $15 \mathrm{~mL}$ Falcon tube identified with a five-digit number bar code and the date of collection.
Cervical samples will be obtained using the Digene HC2 DNA Collection kit (Qiagen) and placed in $1 \mathrm{~mL}$ of Specimen Transport Medium (STM), according to the manufacturer's instructions.

Penile (penile shaft, glans penis/coronal sulcus) and scrotum samples will be obtained using a Dacron swab (Qiagen) previously moistened in sterile saline solution. Collection will be performed by intensely pressing and rubbing the swab in the epithelium via self-collection under supervision of the primary care health professional and after instruction regarding the procedure.

All biological samples will be maintained at room temperature $\left(15^{\circ} \mathrm{C}-25^{\circ} \mathrm{C}\right)$ according to manufacturer's instructions and transported to a central laboratory in Porto Alegre to start the specimen processing. The temperature will be monitored with a data logger. Figure 1 presents the general logistics for the nationwide multicentre collection of data and specimens.

Individuals who present visible lesions on the genitals or in the oral cavity at the time of recruitment will be asked to grant permission for a picture to be taken of the lesion.

\section{Specimen processing}

All samples will be treated as biohazardous material, and all specimen handling will be performed in a biosafety cabinet. In the laboratory, specimens will be immediately aliquoted. One aliquot will be maintained at $-80^{\circ} \mathrm{C}$ as a backup. Another aliquot will be processed for DNA extraction from the STM medium using magnetic beads for isolation and purification using the automatised extraction platform (MagNA Pure LC V.2.0, Roche Molecular Systems), after exposed to an enzymatic treatment with proteinase $\mathrm{K}$, following the manufacturer's instruction.

HPV detection and typing will be done using the Linear Array HPV Genotyping Test (Roche Diagnostics) which is based on PCR amplification ( $450 \mathrm{bp}$ fragment of the polymorphic region of the L1 gene of HPV), followed by specific hybridisation and colorimetric detection, according to the manufacturer's instructions. The test detects HR-HPVs $(16,18,31,33,35,39,45,51,52,56$, 58,59 and 68) and LR-HPVs $(6,11,26,40,42,53,54$, $55,61,62,64,66,67,69,70,71$ 72, 73, 81, 82, 83, 84, IS39 and CP6198), for a total of 37 types. The amplification of human $\beta$-globin (biotinylated primers GH20 and PC04, 268 bp fragment) is used as an internal control for the PCR. To ensure reproducibility of LA, an automated AutoBlot instrument (Fujirebio) was used for hybridisation and wash steps. Real-time PCR using the TaqMan system for HPV type 52 will be performed to confirm the results obtained by Roche's test as a combination of HPV types 52, 33, 35 and 58 .

\section{Data analyses and statistics}

Categorical variables will be summarised using absolute frequencies and percentage, while continuous variables will be analysed using means and SE. $\chi^{2}$ tests and Fisher's 


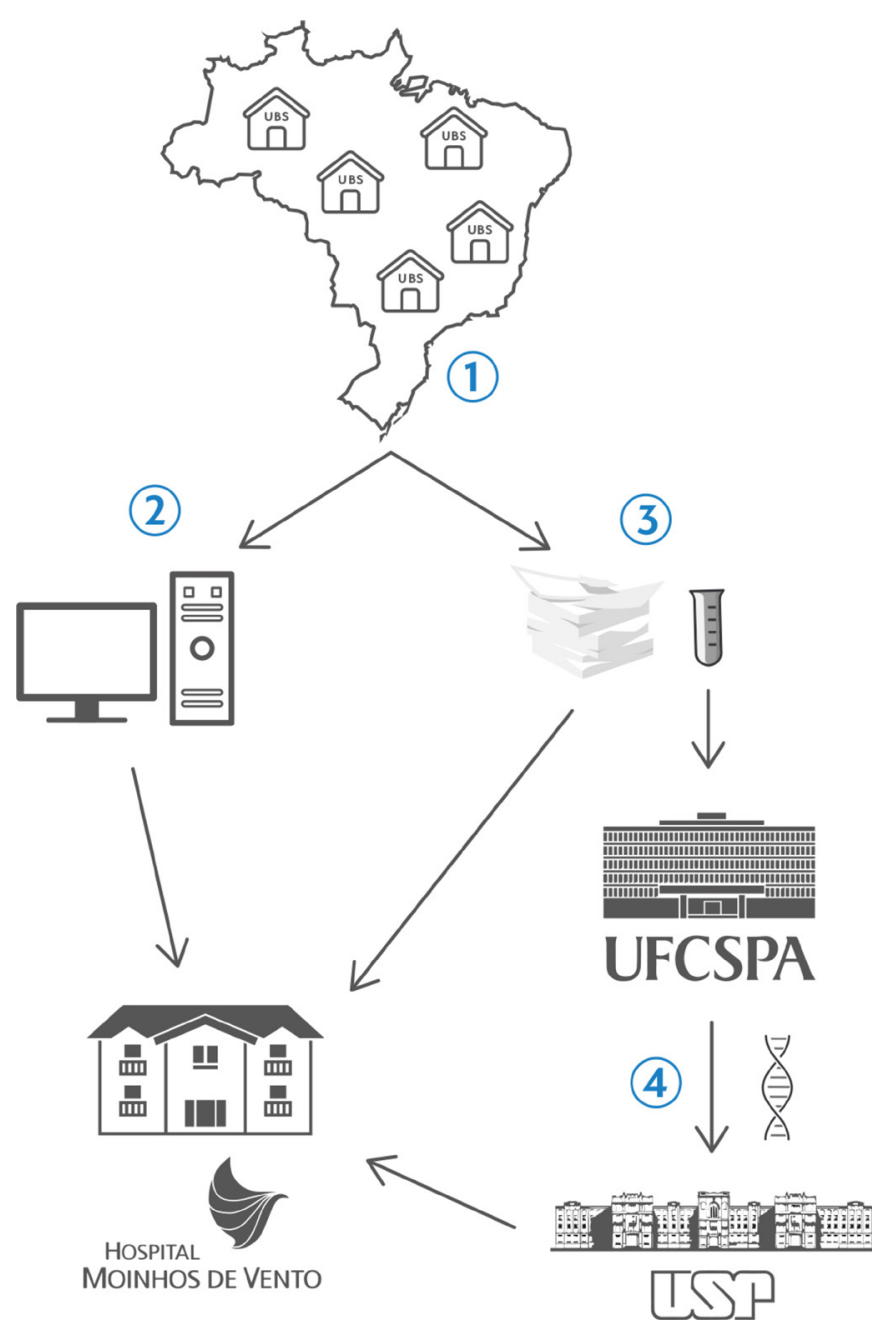

Figure 1 Workflow of the study. (1) Data are collected in public health units from all 27 Brazilian capitals; (2) data entry into the web-based system is performed by primary care professionals and is monitored by the main research team in Porto Alegre (Hospital Moinhos de Vento); (3) paper forms are sent to Porto Alegre along with biological samples which are processed for DNA extraction Universidade Federal de Ciências da Saúde de Porto Alegre (UFCSPA) and (4) extracted DNA is sent to Universidade de São Paulo (USP) for HPV detection and typing. Finally, the results are included in the web-based system and are made available for the primary care professionals and participants using a code number and password. HPV, human papillomavirus.

exact tests will be used to compare proportions, and t-tests or Mann-Whitney non-parametric tests will be used for continuous variables.

The association between HPV infection and social and behavioural variables will be defined by calculating crude and adjusted prevalence ratios. Confounding variables will be evaluated using modified Poisson regression analysis. ${ }^{27}$

To maximise diversity across regions, we have divided the sample size into equal numbers among regions. To adjust the distribution of the sample to the study population, we will standardise the measures by the size of the population in each capital within the age range and by sex.

\section{Monitoring and quality control}

The data quality will be validated by routine practices. A pilot study was performed to identify and correct problems in data collection, sample transportation, procedures and instruments. All health professionals involved in the research are trained and certified by the coordinator team using simulated interview and sample collection according to the study protocol, as described in the POP-Brazil operations manuals.

Data and specimen collection are periodically evaluated by reports generated by the data system, including information about the number of participants and the number of refusals, and the quality of the samples collected. Those reports will be distributed monthly to all people involved in data collection.

Moreover, each unit will receive at least one in loco monitoring visit which will be done by research staff supervisors to evaluate protocol compliance and provide recertification. Supervisors will observe techniques using predefined check lists and recorded interviews.

The biological sample tracking is done through the web-based data platform, and time, temperature and sample conditions are evaluated on a daily basis.

To access the reliability of the interviews, a subsample will be obtained in duplicate. A resampling will be done in $10 \%$ of the interviews using phone calls to access the coefficient of variation and systematic differences between data. Figure 2 presents the steps that will be taken for quality assurance. The reliability of HPV detection will be accessed by double processing a random subsample of the biological genital samples by the Centers for Disease Control and Prevention.

\section{Patient and public involvement}

Patients and public were not involved in the study design and/or protocol. In cases of no infection or infection with a low-risk HPV type, participants will be informed of negative results via web access. Conversely, participants infected with high-risk HPV types will be advised to go to the healthcare unit for their results, where they will be oriented about the meaning of the result and instructed about follow-up according to national guidelines.

Participants will be informed about the study results through study website.

\section{DISCUSSION}

This is the first Brazilian nationwide study to determine HPV prevalence and the social, demographic and behavioural factors related to HPV infection. Critical analysis of the study results will contribute to epidemiological knowledge of the distribution of HPV strains across geographical regions and will establish a baseline to evaluate the impact of the Brazilian Vaccination Program. 


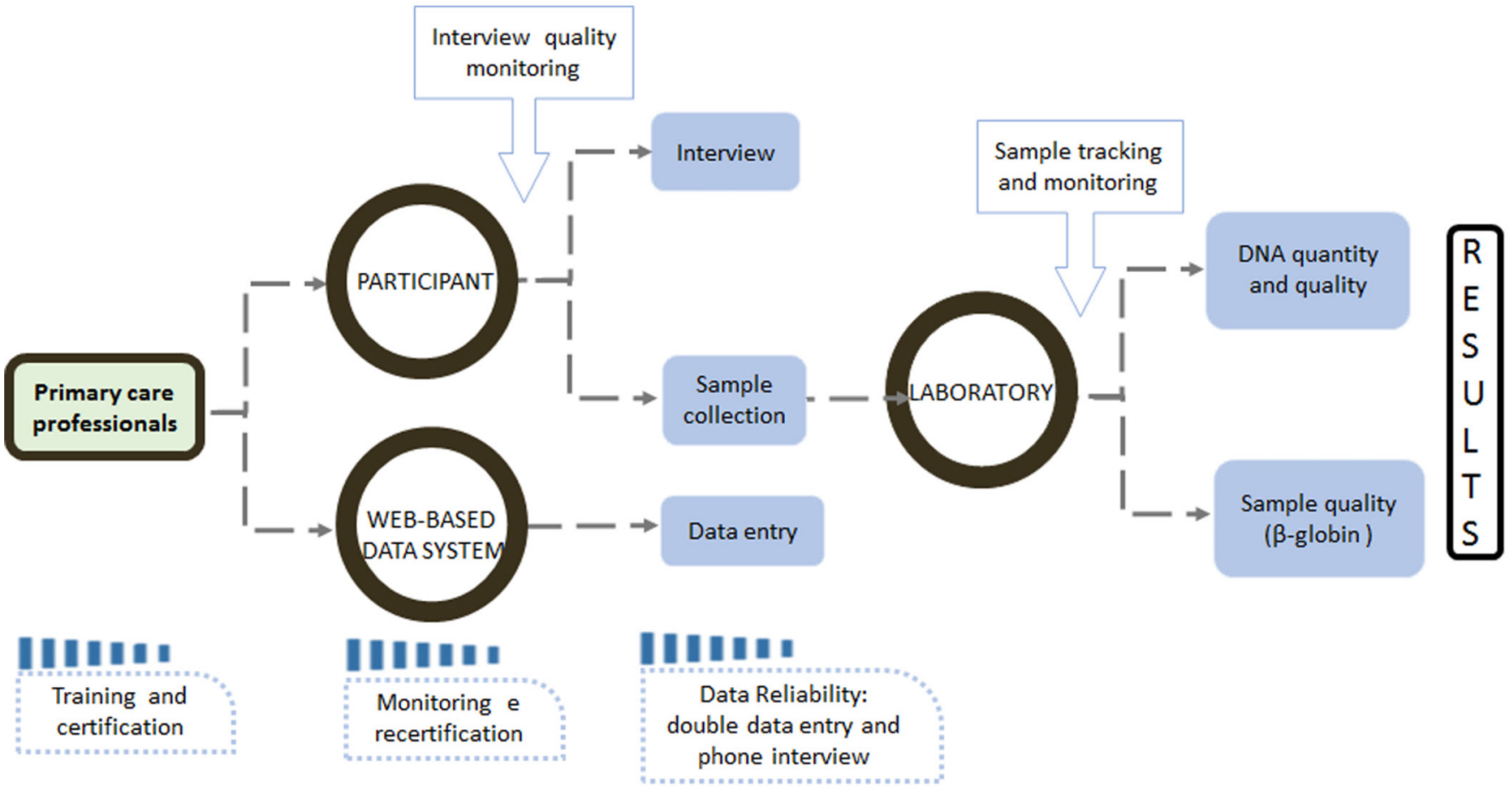

Main researchers team

Figure 2 Description of the study's major quality-control steps during each phase of the study.

There are some limitations to this study. The sampling is not random. Brazil is a large country, and due to logistic reasons, the sampling is restricted to state capitals in public health units selected by the local government. To minimise selection bias, in addition to recruiting participants who voluntarily come to the health unit, we will also recruit at schools, through the list of patients of the selected health units and by personal invitation by community health agents in households. We are also only recruiting participants in the public health system, who account for approximately $70 \%$ of the overall Brazilian population; therefore, we cannot draw inferences about people who exclusively use the private sector. In Brazil, the private sector is mainly used by social classes A and B, but it is important to highlight that for some services such as vaccination, the Public Health System covers virtually $100 \%$ of the population.

The strengths of the study are the establishment of a baseline for future comparison of HPV prevalence, the nationwide sampling, including a large and diverse population and the use of central laboratory and techniques for data quality monitoring. Additionally, we will collect data on sexual behaviours and other STIs, making it possible to pursue some explanation regarding differences in prevalence according to specific groups of participants.

Health managers, based on these data, will be able to compare HPV prevalence in different regions according to population characteristics. It will be possible to support the development of strategies to promote HPV vaccination and to develop strategies for prevention, taking into account regional differences. Moreover, these data will serve as a baseline for future comparisons and will allow monitoring of the effectiveness of the National HPV Vaccination Program.

\section{ETHICS AND DISSEMINATION}

Before starting the study, this project was submitted to the research ethical committee of the proposing institution (Hospital Moinhos de Vento). Following approval, agreements were made by all coparticipant centres and submitted to their corresponding ethical committees.

Individuals must voluntarily agree to participate and must sign the free and informed consent form. The study follows the ethical recommendations of Resolutions 347/05, 441/11 and 466/12 of the National Health Council.

Preliminary results will be presented as poster and/or oral sections in national and international congresses, and the final results will be submitted for publishing in peer-reviewed open-access journals. The results will also be presented in a personal meeting to the Ministry of Health of Brazil.

Author affiliations

${ }^{1}$ Hospital Moinhos de Vento, Porto Alegre, Brazil 
${ }^{2}$ Community Health Department, Federal University of Health Science of Porto Alegre, Porto Alegre, Brazil

${ }^{3}$ National Immunization Program, Brazilian Ministry of Health, Brasília, Brazil ${ }^{4}$ Department of Surveillance, Prevention and Control of Sexually Transmitted Infections, HIV/AIDS and Viral Hepatitis, Brazilian Ministry of Health, Brasília, Brazil ${ }^{5}$ National Institute for Health Technology Assessment, Federal University of Rio Grande do Sul, Porto Alegre, Brazil

${ }^{6}$ Department of Preventive and Social Odontology, Federal University of Rio Grande do Sul, Porto Alegre, Brazil

${ }^{7}$ Universidade de São Paulo and Instituto do Cancer do Estado de São Paulo (ICESP), Sao Paulo, Brazil

Acknowledgements The authors would like to thank Julian Peto and Claire Gilham from the London School of Hygiene and Tropical Medicine for helping with the development of sampling procedures and Elisabeth Unger and Lauri Markowitz from the Centers for Disease Control and Prevention for providing helpful suggestions in developing the study protocol and quality control.

Contributors EMW conceived of the project and participated in all phases of the manuscript preparation; ASB, AGKM, LSH, FMAdS, JBH, FNH, MF, LLV, CD and MB participated in the protocol development; and JC and LLV participated in the laboratory protocol development and helped write the manuscript. All authors reviewed the final version of the manuscript.

Funding This work is financed by Hospital Moinhos de Vento through the Program for Supporting the Institutional Development of the Public Health System (PROADI-SUS), supported by the Ministry of Health of Brazil, with funding participation of the Pan-American Health Organization.

Competing interests ASB, FMAdS, CD and AGKM work for the Ministry of Health of Brazil. LLV is a consultant for Merck for the HPV quadrivalent vaccine and for Qiagen, BD and Roche for HPV DNA tests.

Patient consent Obtained.

Ethics approval Research Ethical Committee of Moinhos de Vento Hospital. Provenance and peer review Not commissioned; externally peer reviewed.

Open Access This is an Open Access article distributed in accordance with the Creative Commons Attribution Non Commercial (CC BY-NC 4.0) license, which permits others to distribute, remix, adapt, build upon this work non-commercially, and license their derivative works on different terms, provided the original work is properly cited and the use is non-commercial. See: http://creativecommons.org/ licenses/by-nc/4.0/

(C) Article author(s) (or their employer(s) unless otherwise stated in the text of the article) 2018. All rights reserved. No commercial use is permitted unless otherwise expressly granted.

\section{REFERENCES}

1. Globocan. Home. 2012. http://globocan.iarc.fr/Default.aspx (accessed 1 Dec 2017).

2. Walboomers JM, Jacobs MV, Manos MM, et al. Human papillomavirus is a necessary cause of invasive cervical cancer worldwide. J Pathol 1999;189:12-19.

3. Bosch FX, Lorincz A, Muñoz N, et al. The causal relation between human papillomavirus and cervical cancer. J Clin Pathol 2002:55:244-65.

4. Guan P, Howell-Jones R, Li N, et al. Human papillomavirus types in 115,789 HPV-positive women: a meta-analysis from cervical infection to cancer. Int J Cancer 2012;131:2349-59.

5. Portal-Instituto Nacional de Câncer-INCA. INCA promove exposição sobre o câncer do colo do útero. http://www2.inca.gov.br/wps/wcm/ connect/inca/portal/home (accessed 1 Dec 2017).
6. Serrano B, Brotons M, Bosch FX, et al. Epidemiology and burden of HPV-related disease. Best Pract Res Clin Obstet Gynaecol 2018; $47: 14-26$

7. Han JJ, Tarney CM, Song J. Variation in genital human papillomavirus infection prevalence and vaccination coverage among men and women in the USA. Future Oncol 2017;13:1129-32.

8. Taberna M, Mena M, Pavón MA, et al. Human papillomavirus-related oropharyngeal cancer. Ann Oncol 2017;28:2386-98.

9. Centers for Disease Control and Prevention. HPV | Home | Human Papillomavirus | CDC. https://www.cdc.gov/hpv/index.html (accessed 1 Dec 2017).

10. Syrjänen K, Hakama M, Saarikoski S, et al. Prevalence, incidence, and estimated life-time risk of cervical human papillomavirus infections in a nonselected Finnish female population. Sex Transm Dis 1990;17:15-19.

11. HPV Information centre. http://www.hpvcentre.net/ (accessed 1 Dec 2017).

12. Colpani V, Bidinotto AB, Falavigna M, et al. Prevalence of papillomavirus in Brazil: a systematic review protocol. BMJ Open 2016;6:e011884.

13. Coser J, Boeira TR, Wolf JM, et al. Cervical human papillomavirus infection and persistence: a clinic-based study in the countryside from South Brazil. Braz J Infect Dis 2016;20:61-8.

14. de Oliveira GR, Vieira VC, Barral MF, et al. Risk factors and prevalence of HPV infection in patients from Basic Health Units of an University Hospital in Southern Brazil. Rev Bras Ginecol Obstet 2013;35:226-32.

15. Corrêa CM, Teixeira NC, Araújo AC, et al. Prevalence and multiplicity of HPV in HIV women in Minas Gerais, Brazil. Rev Assoc Med Bras 2011:57:425-30.

16. da Silva MC, Martins HP, de Souza JL, et al. Prevalence of HPV infection and genotypes in women with normal cervical cytology in the state of Paraná, Brazil. Arch Gynecol Obstet 2012;286:1015-22.

17. Girianelli VR, Thuler LC, e Silva GA. Prevalence of HPV infection among women covered by the family health program in the Baixada Fluminense, Rio de Janeiro, Brazil. Rev Bras Ginecol Obstet 2010;32:39-46.

18. Meyrelles AR, Siqueira JD, Hofer CB, et al. HIV/HPV co-infection during pregnancy in southeastern Brazil: prevalence, HPV types, cytological abnormalities and risk factors. Gynecol Oncol 2013;128:107-12.

19. Silva LM, Santos WG, Santiago MB. Prevalence of cutaneous warts in patients with systemic lupus erythematosus: a systematic review. $J$ Infect Dev Ctries 2016;10:902-6.

20. Vieira RC, Monteiro JS, Manso EP, et al. Prevalence of type-specific HPV among female university students from northern Brazil. Infect Agent Cancer 2015;10:21.

21 HPV |UNA-SUS. https://www.unasus.gov.br/noticia/meninoscome\%C3\%A7am-ser-vacinados-contra-hpv-na-rede-p\%C3\% BAblica-de-sa\%C3\%BAde (accessed 1 Dec 2017).

22. Ayres ARG, Silva GA. Cervical HPV infection in Brazil: systematic review. Rev Saúde Pública 2010;44:963-74.

23. Saulle R, Miccoli S, Unim B, et al. Validation of a questionnaire for young women to assess knowledge, attitudes and behaviors towards cervical screening and vaccination against HPV in Italy. Epidemiol Biostat Public Health 2014;11.

24. Critério Brasil - ABEP. Critério Brasil 2018. http://www.abep.org/ criterio-brasil (accessed 1 Dec 2017).

25. Soares AHR. O aprendizado da sexualidade: reprodução e trajetórias sociais de jovens brasileiros. Cadernos de Saúde Pública 2007;23:1495-6.

26. Comportamento sexual da população brasileira e percepções do HIV/Aids | Biblioteca Virtual em Saúde. http://pesquisa.bvsalud.org/ bvsms/resource/pt/mis-3389 (accessed 1 Dec 2017).

27. Barros AJ, Hirakata VN. Alternatives for logistic regression in crosssectional studies: an empirical comparison of models that directly estimate the prevalence ratio. BMC Med Res Methodol 2003;3:21. 